Strontium werden mitgeteilt. Die experimentellen Daten für diese Berechnungen werden weitgehend chromatographisch ermittelt. Der Verfasser erwähnt, daß er Versuche angestellt hat, die Größe $n_{s}$ exakt zu bestimmen, um exaktere Werte für die thermodynamischen Funktionen zu erhalten.

\section{Lösliche Kieselsäure in Wasserstoff-Beidellit}

Woodruff, C. M., University of Missouri, Columbia, Missouri.

Elektrodialysierte Suspensionen von Tonmineralien werden im allgemeinen als unlösliche Silikate angesehen, in denen der Austauschkomplex mit Wasserstoff gesättigt ist. Eine Untersuchung der relativen Aktivitäten verschiedener austauschfähiger Ionen an Beidellit-Ton ergab die Tatsache, daß ein frisch zubereiteter Wasserstoff-Ton, wenn er eine Spur von nichtabsorbiertem Chlorion enthält, beim Filtrieren eine Lösung lieferte, in der das Chlorion nicht mit dem Wasserstoff verbunden war, sondern vielmehr mit der Kieselsäure.

Das Filtrat von der frisch zubereiteten Suspension war klar und hatte ein $\mathrm{p}_{\mathrm{H}}$ von 5,5. Nach 24 stündigem Stehen wurde das Filtrat trübe und der $\mathrm{p}_{\mathrm{H}}$-Wert fiel auf 3,5, was der Bildung von Säure aus Chlorion und Wasserstoff entspricht. Analyse der klaren Lösung ergab, daß die Chloride mit der Kieselsäure verbunden vorliegen. Der Anteil an löslicher Kieselsäure im Filtrat von der Ton-Suspension läßt sich unmittelbar auf den Anteil austauschbaren Wasserstoffs im Ton beziehen. Keine lösliche Kieselsäure fand sich dagegen im Filtrat von Ton vor, der mit Kalium- oder Calciumhydroxyd neutralisiert worden war.

Die Resultate führen zu dem Schluß, daß die Tonschichten, die für manche saure Böden charakteristisch sind, dadurch gebildet wurden, daß die austauschbaren Basen im Laufe der Verwitterung durch Wasserstoff ersetzt werden.

\section{Ein amerikanisches Vorkommen von Wolchonskoit}

McConnell, Duncan, College of Engineering, The Ohio State University, Columbus, Ohio.

Gewisse zutage tretende Oberflächenschichten der Morrison-Formation bei Thompson im Staate Utah sind intensiv grün, und zwar entlang von Rißspalten im Sandstein.
Das färbende Agens kann von anderen Bestandteilen des bröckligen Gesteins durch Schlämmen des zermahlenen Materials abgetrennt werden. Auf Grund der großen Ähnlichkeit des Röntgenogramms dieses grünen Minerals mit dem des Nontronits und auf Grund seines Gehalts von ungefähr $2 \% \mathrm{Cr}_{2} \mathrm{O}_{3}$ konnte es als Wolchonskoit identifiziert werden.

Bislang wurde dieses chromhaltige Glied der Montmorillonit-Nontronit-Reihe nur auf russischen Fundstellen (Ural) angetroffen.

Bewertung von Methoden für die quantitative Analyse von Halloysit-Kaolinit-Tonen

Sand, L. B. und W. C. Ormsby, School of Mineral Industries, The Pennsylvania State College, State College, Pennsylvania, und Standard Oil Company, Research Department, Whiting, Indiana.

Eine Anzahl von Untersuchungsmethoden wurde geprüft hinsichtlich ihrer Anwendbarkeit auf das Problem, den Gehalt an Halloysit in Kaolin-Tonen zu bestimmen. Diese Methoden umfassen Dünnschliffuntersuchungen, Bestimmungen des Basenaustausches, elektronenmikroskopische und Ultrarotabsorptions-Messungen.

Basenaustauschbestimmungen wurden an einer Anzahl von monodispersen Fraktionen reiner Halloysite and Kaolinite durchgeführt. Für Halloysite und Kaolinite der Teilchengröße $1-0,25 \mu$ ergaben sich als konsistente Werte 12 bzw. 4 Milliäquivalente/100 g.

Elektronenmikrogramme wurden von homogenisierten Gemischen angefertigt and Auszählungen der zwei morphologisch verschiedenen Varietäten vorgenommen. Anwendung dieser Untersuchungstechnik auf einige natürliche halloysithaltige Kaoline, deren Mengenanteile durch Differentialthermoanalyse festgestellt waren, gaben Resultate, die sich mit denen aus der DTA deckten.

Die Dünnschliff-Methode gab sehr gute Ergebnisse bei Tonen auf primärer Lagerstätte, wo der Kaolinit als wurmförmige Aggregate vorkommt.

Infrarot-Absorptionsspektren von künstlichen Gemischen wurden durchgemessen; die Unterschiede für Halloysit und Kaolinit waren genügend ausgeprägt, so daß halbquantitative Ergebnisse zu erhalten waren.

Diese vier Methoden wurden hinsichtlich Genauig. keit und Anwendbarkeit kritisch geprüft und mit anderen empfohlenen Techniken der Untersuchung verglichen.

\title{
Berichtigung
}

\section{Electron Microscope Studies on the Aging of Amorphous Colloidal Aluminium Hydroxide}

Von P.Souzo Santos, Vallejo-Freire,A. und So uzo Santos, H. L.-Sao Paulo/Bras. Kolloid-Z. 133, 101 (1953)

Auf Seite 107, 21. Zeile von unten, linke Spalte, muß es statt „,tactoids“", ,somatoids“ heißen. 